\title{
Optical seeing monitoring at the Oukaïmeden in the Moroccan high atlas mountains: first statistics
}

\author{
Z. Benkhaldoun, A. Abahamid, Y. El Azhari, and M. Lazrek
}

\author{
Laboratoire de Physique des Hautes Energies et Astrophysique, Département de Physique, Faculté des Sciences Semlalia, \\ BP 2390, Marrakech, Maroc \\ e-mail: zouhair@ucam.ac.ma
}

Received 10 December 2004 / Accepted 19 May 2005

\begin{abstract}
In order to complete characterization of the Oukaïmeden site and hoping to prospect other sites in the Moroccan High Atlas, we constructed a seeing monitor. We named the instrument DIMMAR for Differential Image Motion Monitor of Marrakesh. After more than one year of operation, we can conclude that the DIMMAR is quite reliable and that it can be a prototype for other similar low-cost instruments. In this work, the seeing measured at Oukaïmeden site using the DIMMAR on top of a 5 meters high tower is presented. Statistics from fifteen months seeing's are treated (from July 19, 2003 up to September 26, 2004). The median and mean zenith seeing for the entire period of observations are $0.84^{\prime \prime}$ and $0.92^{\prime \prime}$, respectively, at $0.5 \mu \mathrm{m}$ (or $0.96^{\prime \prime}$ and $1.05^{\prime \prime}$, if corrected for exposure-time bias). The best seeing value measured is $0.32^{\prime \prime}$.
\end{abstract}

Key words. site testing - turbulence - methode: data analysis

\section{Introduction}

The renewed interest in ground-based astronomy and the developing worldwide search for sites with good seeing for the next generation of the Extremely Large Telescopes (ELT), led us to develop an instrument to monitor astronomical seeing. The possible selection of the Moroccan High Atlas mountains as a potential site for ELT's encouraged us to install this instrument at Oukaïmeden for testing its capability and to complete the qualification studies of this site as a first candidate in the High Atlas site search. Our plan is to build several copies of this instrument and set them in different locations to assist us in the next site prospection campaigns.

Oukaïmeden site is located at $2700 \mathrm{~m}$ above sea level $\left(7^{\circ} 52^{\prime} 52^{\prime \prime} \mathrm{W}, 31^{\circ} 12^{\prime} 32^{\prime \prime} \mathrm{N}\right), 70 \mathrm{~km}$ from Marrakesh city. The procedure of selection of the Oukaïmeden as a potential site for astronomical observations was presented by Benkhaldoun et al. (1991) and Benkhaldoun (1994). The studies of photometric and meteorological qualification were also treated in several publications (Benkhaldoun 1993; Hill 1994a,b; Jabiri et al. 2000; Benkhaldoun 2002). Measurements of seeing were also carried out by the GSM instrument, but over a very short period (11 days in 1998). Ziad et al. (2001) published the main results of this campaign, which conclude that further measurements over a long period must be performed to study the seeing quality at Oukaïmeden.

Accurate, precise, and reproducible measurements are the qualities expected of any instrument. The Differential Image Motion Monitor of Marrakesh (DIMMAR) is presented to assess astronomical seeing with those requirements. It has also been designed to be reliable, simple, transportable, inexpensive, and easy to set up, operate, and maintain. Its accuracy has been studied, both from theoretical and experimental points of view, mainly with respect to CCD (Charge Coupled Device) sampling manufactured by SBIG (Santa Barbara Instrument Group), photon noise, and threshold influence on centroid determination.

A long duration measurement campaign was performed at the Oukaïmeden site with DIMMAR. The DIMM principle, DIMMAR characteristics and the measurement campaign are presented in Sect. 2. The results of atmospheric optical parameters with DIMMAR are presented in Sect. 3 and the results discussed in Sect. 4.

\section{The DIMMAR instrument}

\subsection{DIMM principle}

Turbulence in the Earth's atmosphere causes the stellar image formed by a telescope to move randomly. The position of the stellar image in the focal plane is directly related to the tilt angle of the wavefront with regard to the entrance pupil plane of the telescope. According to theoretical calculations, the atmospheric turbulence can be estimated through the variance of the image motion. The DIMM method consists of measuring the differential motion of two stellar images at the focus of a small telescope. A mask with two sub-apertures is located at the entrance pupil. One of the sub-apertures contains a prism, so that two images of the same star are observed at the focus. The dual star images obtained exhibit a relative motion in the 
image plane that represents the local wavefront tilts, which can be expressed in terms of an absolute seeing scale according to the approximate formula (Roddier 1981; Sarazin 1990; Dierickx 1992), where the seeing is described in terms of the full width at half-maximum intensity $(F W H M)$ :

$F W H M=0.976 \frac{\lambda}{r_{\mathrm{o}}}$

where $\lambda$ is the wavelength and $r_{\mathrm{o}}$ is the Fried parameter.

For a given wavefront of coherence radius $r_{\mathrm{o}}$, the variance of the differential motion (in radian ${ }^{2}$ ) between two pupils of diameter $D$ at the distance $d$ of each other depends on the direction of measurement (which holds when $D / d \leq 0.5$, Sarazin \& Roddier 1990):

$\sigma_{\|}^{2} \simeq 2\left(0.179 D^{-1 / 3}-0.0968 d^{-1 / 3}\right) \lambda^{2} r_{\mathrm{o}}^{-5 / 3}$

$\sigma_{\perp}^{2} \simeq 2\left(0.179 D^{-1 / 3}-0.145 d^{-1 / 3}\right) \lambda^{2} r_{\mathrm{o}}^{-5 / 3}$

where $\sigma_{\|}$is the variance of the differential longitudinal motion and $\sigma_{\perp}^{2}$ the variance of the differential transverse motion.

We note that direction-dependent sensitivity of the image motion to changes in seeing quality is given by the constant coefficients $\left(K=\frac{\sigma^{2}}{\lambda^{2} r_{0}^{-5 / 3}}\right)$ :

$K_{\|}=2\left(0.179 D^{-1 / 3}-0.0968 d^{-1 / 3}\right)$

$K_{\perp}=2\left(0.179 D^{-1 / 3}-0.145 d^{-1 / 3}\right)$.

Finally the site quality defined as the long exposure $F W H M$ (in radian) at the wavelength $\lambda$ and at zenith is related to the variance of the image motion measured in either direction $*$ at zenith angle $\gamma$ by:

$F W H M_{*}=0.976 \lambda^{-1 / 5}\left[\frac{\sigma_{*}^{2} \cos \gamma}{K_{*}}\right]^{3 / 5}$.

To improve the accuracy, two seeing measurements are made at present: a "longitudinal" one in the direction parallel (\|) to the sub-aperture axis, and the "transverse" one that is perpendicu$\operatorname{lar}(\perp)$. The final result is the average of both at $\lambda=0.5 \mu \mathrm{m}$ expressed in arcsec.

\subsection{The DIMMAR technical parameters}

The DIMMAR instrument monitored different parameters of the atmospheric turbulence, such as Scintillation Index, Extinction coefficient and Seeing. The technique employed is based on statistical analysis of the differential tilt of the wavefront at two locations $14 \mathrm{~cm}$ apart. The standard deviation of the differential slope of 100 short exposures with an integration time $10 \mathrm{~ms}$ is converted into the FWHM of a long exposure stellar image by means of the appropriate equations above (6). The measures of the seeing change over a time interval $\Delta t=100 \mathrm{~s}$. This requires short exposures at a few milli-second intervals, which can be obtained using either a fast read-out camera or non-destructive partial frame transfer.

The components of the DIMMAR are as follows (Fig. 1, Table 1). The laptop is connected to the parallel port of the

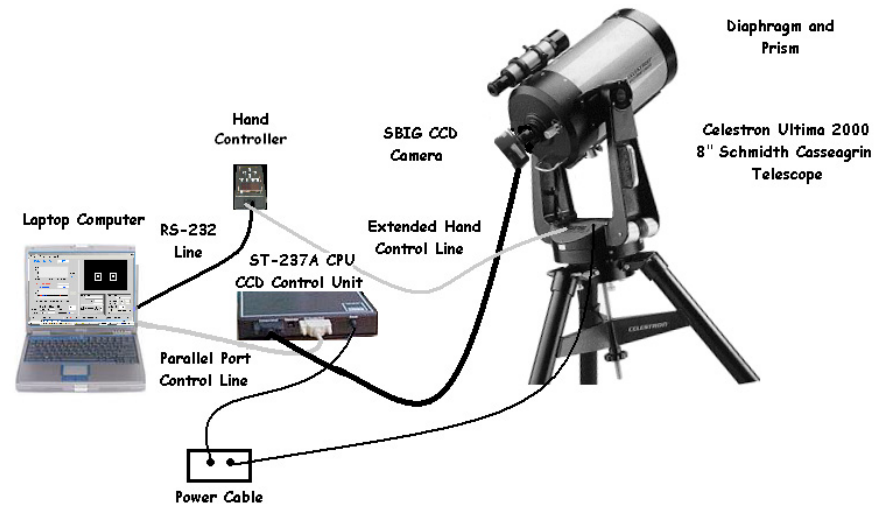

Fig. 1. General configuration and components of the DIMMAR.

Table 1. The DIMMAR technical parameters.

\begin{tabular}{ll}
\hline \hline Telescope type & $8^{\prime \prime}$ Ultima 2000 Celestron \\
\hline Telescope Diameter & $203 \mathrm{~mm}$ \\
Telescope Focal Length & $2032 \mathrm{~mm}$ \\
Twin Pupil Diameter & $60 \mathrm{~mm}$ \\
Distance Between Pupils & $140 \mathrm{~mm}$ \\
Prism Deviation Angle & $40 \mathrm{arcsec}$ \\
Camera model & ST-237A SBIG CCD \\
Pixel Array & $657 \times 495$ pixels, $4.9 \times 3.7 \mathrm{~mm}$ \\
Pixel Size & $7.4 \times 7.4$ microns $(0.73 \mathrm{arcsec})$ \\
\hline
\end{tabular}

CCD control unit. The communication with the telescope is established via RS-232 serial port at the laptop and the 6-line phone jack connector of the telescope via its hand controller. These instruments use a cheap available telescope and CCD camera. To avoid surface layer influence (Vernin \& Muñoz-Tuñón 1994), the DIMMAR was located on a 5-m tower, constructed especially for this experiment.

DIMMAR operation consists of pointing at a star within $30^{\circ}$ of zenith, which allows reduction to the zenith with sufficient accuracy. The star selected is of visual magnitude from 0 to 3 . From the CCD frame, only two subarrays of $20 \times 20$ pixels are digitized and transferred to the computer through a 16-bit parallel interface. The centers of images formed by subapertures are referred to centers of gravity. We used the windowing method (Tokovinin 2002). The centroids are then computed within the subarrays. The uncertainty in the determination of the relative positions of the image centroids corresponds to a noise of variance: $\sigma_{\mathrm{n}}^{2}=(6 \pm 3) 10^{-4} \mathrm{arcsec}^{2}$, as measured in the laboratory on two fixed spots. It was subtracted from the measured variance of differential image motion in DIMM to remove the bias due to readout noise. We use the centroids separations ( $\mathrm{dx}$ and $\mathrm{dy}$ ) to control the correct focus and telescope alignement.

Equivalent long-exposure seeing is computed from the rms differential image motion in the direction of the aperture separation (longitudinal) and perpendicular to the aperture separation (transverse) estimated every 100 seconds from series of 100 frames of $\tau=10 \mathrm{~ms}$. This low data rate is due to the limited speed of transfer in the parallel link between the CCD Control Unit (CPU) and the computer (Fig. 1). As a consequence, the statistical error on the image motion variance estimate is 
the dominating noise term, on the order of $\pm 1 /(100-1)^{1 / 2}$ or $\pm 10 \%$. This random error is fortunately averaged out in long term statistics. The images are not stored, but processed immediately, and only arrays of coordinate differences, flux, and air mass are accumulated. This array is used to compute the coordinate difference variance (e.g. $F W H M$ ), mean flux, and scintillation. Finally, all reduced data are saved.

The variances are obtained in unit of pixel square and require a calibration of the pixel size. That was done by imaging the double stars (like Mizar, which angular separation $\rho=14.5$ arcsec). The measured angular size of the pixel is 0.79 arcsec. A pixel scale computed from nominal telescope focus is about 0.73 arcsec. We used the measured angular size for our seeing determination.

The finite exposure time can biase seeing to better values. The interlacing method proposed by Tokovinin (2002) estimates this bias as from $10 \%$ to $20 \%$. Results of seeing presented here are for a finite exposure time of $10 \mathrm{~ms}$. However, we made some measurements, using interlaced method with our equipment, and found a correction factor of about $14 \%$, estimated from interlaced series of 100 individual frames of $10 \mathrm{~ms}$ and $20 \mathrm{~ms}$. This correction factor may be variable in time, but it gives an idea of a potential bias in our data. We present the results without this correction, unless stated otherwise.

\section{First statistical results of DIMMAR measurements made at Oukaïmeden site}

The available data covers the nights between 19 July 2003 and 26 September 2004. They are presented in the figures and tables shown below. Ideally, one data file is generated per observed star. Each line of the data file corresponds to a seeing measurement including: universal time, longitudinal $\left(F W H M_{\|}\right)$, transverse $\left(F W H M_{\perp}\right)$, mean $\left(\left(F W H M_{\|}+F W H M_{\perp}\right) / 2\right)$, and ratio $\left(F W H M_{\|} / F W H M_{\perp}\right)$ seeing, flux, scintillation index, and airmass $(1 / \cos \gamma)$. We only considered the stars close to the zenith (airmass $\leq 1.15$ ).

The two estimations of seeing (longitudinal and transverse) are expected to give similar values; all measurements with $\mid 1-$ $\varepsilon_{l} / \varepsilon_{t} \mid>0.12$ were rejected (Muñoz-Tuñón et al. 1997; Aristidi et al. 2002).

The seeing and the scintillation index have been calculated and have compensated for the zenith angle effect. In practice the working conditions are such that interruptions, such as bad weather, often force a power cut and observer absence.

\subsection{Daily statistics at Oukaïmeden site}

The temporal evolution of the measurements provided at night $07 / 25 / 2003$ are presented in Fig. 2 at zenith and $0.5 \mu \mathrm{m}$. The ratio of longitudinal to transverse seeing values is given versus time. This ratio being always around unity, it ensures the reliability of the measurements.

The seeing value time series shown in Fig. 2 indicate that small variations may occur during a whole night and that the seeing obtained with longitudinal and transverse analysis are quite similar (i.e. ratio $\simeq 1$ ). The mean and median zenith

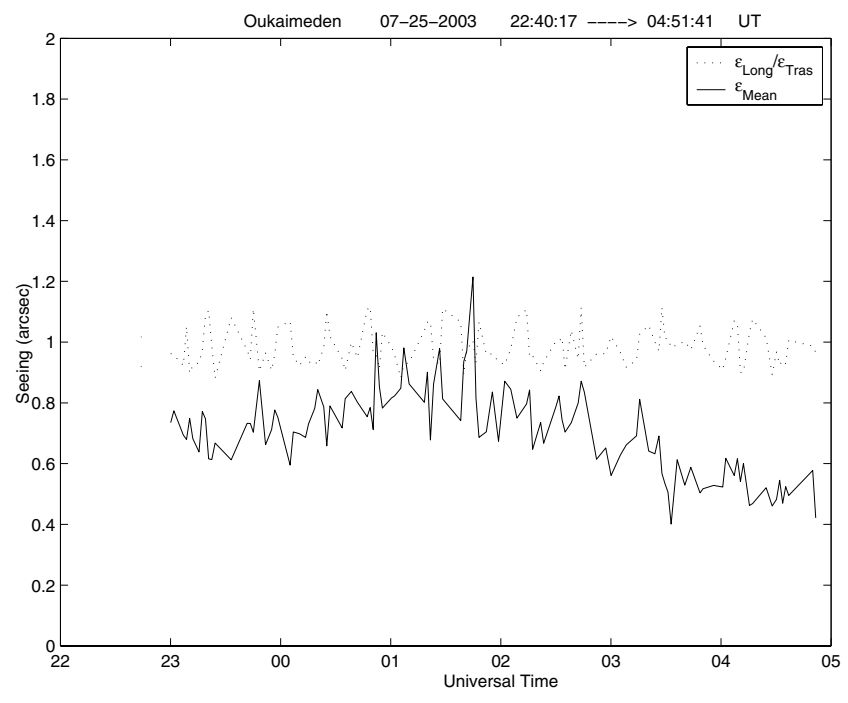

Fig. 2. The temporal evolution of the longitudinal to transverse seeing ratio and mean seeing at Oukaïmeden site during the whole night of 25 to $26 / 07 / 03$.

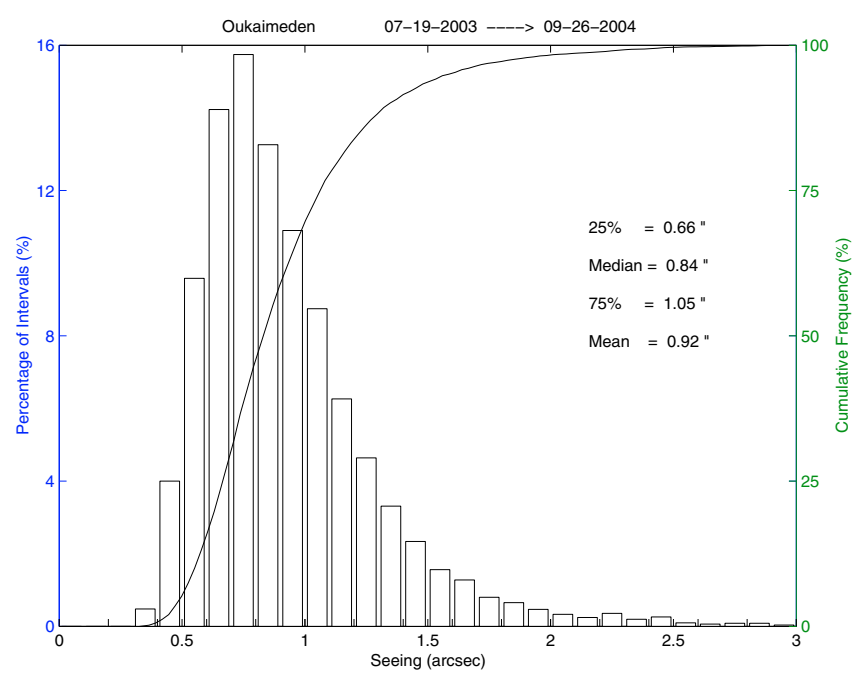

Fig. 3. Statistical distribution of Oukaïmeden seeing during the whole period of observation.

seeing for the entire night are $0.70^{\prime \prime}$, with a narrow distribution $\left(\mathrm{rms}=0.14^{\prime \prime}\right)$.

The seeing statistics obtained from July 2003 to September 2004 are presented in Figs. 3-4 and Table 2. The histogram of seeing values and cumulative frequency during 137 nights of the campaign is given in Fig. 3.

The relatively low output rate of the DIMMAR is explained primarily by the manpower constraint at Oukaimeden. Indeed on the site there is only one permanent observer, which makes it difficult to continuously monitor over all the observable periods. We are trying to improve this by starting an automatic procedure for the next DIMMAR version.

\subsection{Monthly statistics of DIMMAR measurements}

We analyze here the monthly variation, discarding the months with less than 5 nights of observation (from April to July 2004). 


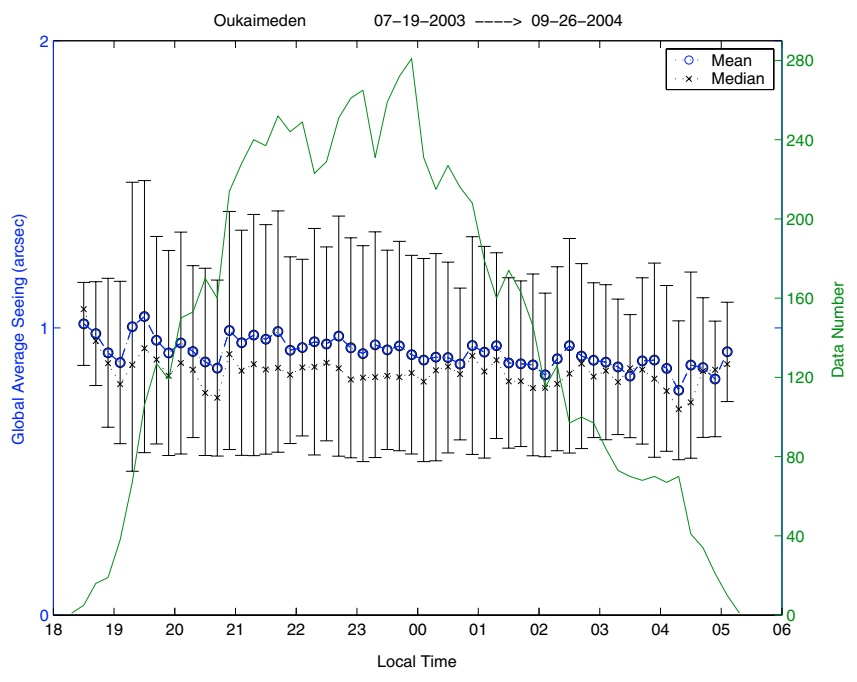

Fig. 4. Averaged seeing versus universal time for the entire observing period.

Table 2. Monthly statistics of the seeing at Oukaïmeden site for the period July 2003 to September 2004. For each month the number of nights and number of seeing measurements are given. The mean, standard deviation, and median values of seeing are presented.

\begin{tabular}{clccccc}
\hline \hline Year & Month & $\begin{array}{c}N \\
\text { nights }\end{array}$ & $\begin{array}{c}N \\
\text { data }\end{array}$ & $\begin{array}{c}\varepsilon_{\text {Mean }} \\
\text { arcsec }\end{array}$ & $\begin{array}{c}S \text { td } \\
\text { arcsec }\end{array}$ & $\begin{array}{c}\varepsilon_{\text {Median }} \\
\text { arcsec }\end{array}$ \\
\hline 2003 & Jul. & 8 & 714 & 0.72 & 0.18 & 0.69 \\
2003 & Aug. & 13 & 777 & 0.85 & 0.27 & 0.79 \\
2003 & Sep. & 12 & 650 & 0.65 & 0.21 & 0.60 \\
2003 & Oct. & 5 & 100 & 1.03 & 0.56 & 0.79 \\
2003 & Nov. & 10 & 844 & 1.00 & 0.42 & 0.89 \\
2003 & Dec. & 18 & 1739 & 0.89 & 0.26 & 0.85 \\
2004 & Jan. & 15 & 1235 & 0.85 & 0.29 & 0.78 \\
2004 & Feb. & 7 & 273 & 0.85 & 0.25 & 0.81 \\
2004 & Mar. & 10 & 279 & 1.28 & 0.44 & 1.23 \\
2004 & Aug. & 19 & 1003 & 1.09 & 0.31 & 1.03 \\
2004 & Sep. & 11 & 373 & 1.17 & 0.46 & 1.01 \\
\hline All & 15 & 137 & 8130 & 0.92 & 0.35 & 0.84 \\
\hline
\end{tabular}

The results of eleven months of measurements are presented in Table 2. For each month, the statistical parameters obtained from the seeing value distribution are calculated. The monthly trend of mean, median, and the standard deviation is shown. In order to give an idea of the statistical significance of the values, the total number of observations made every month with the DIMMAR is presented. The median and mean values of the seeing for the entire period of observation are $0.84^{\prime \prime}$ and $0.92^{\prime \prime}$, respectively, and the standard deviation was $0.35^{\prime \prime}$. The best seeing measured is $0.32^{\prime \prime}$.

In order to compare Oukaimeden to the other mountain sites in terms of seeing, we present some seeing value in Table 3 derived from different instruments at some astronomical sites. $F W H M$ is the mean seeing in arcseconds. All values are corrected to the zenith and at a wavelength of $5 \mu \mathrm{m}$.
Table 3. Mean astronomical seeing in some astronomical observatory over the world. For Oukaimeden, we present here the bias-corrected value of mean seeing.

\begin{tabular}{lccl}
\hline \hline Site & $F W H M$ & Months & References \\
\hline La Palma & 0.76 & 9 & Muñoz-Tuñón et al. (1997) \\
Cerro Paranal & 0.81 & 143 & Sarazin et al. (2003) \\
Mauna Kea & 0.7 & 12 & N.A.O. (2003) \\
La Silla & 0.93 & 158 & Sarazin et al. (1999) \\
Maidanak & 0.76 & 27 & Ehgamberdiev et al. (2000) \\
\hline Oukaïmeden & $\mathbf{1 . 0 5}$ & 15 & This paper \\
\hline
\end{tabular}

\subsection{Temporal dependence}

We have analyzed the temporal variation of the seeing in order to have better insight into diurnal atmospheric behavior that gives rise to optical turbulence, i.e. to know what the typical time interval of seeing variation is, and also the dependence of seeing quality with time, if any.

In Fig. 4 the result of averaging all observing nights is shown versus universal time and a slight trend in the seeing evolution is observed. The seeing is slightly worse at the beginning of the night.

\section{Conclusion}

Statistics of 15 months of seeing at Oukaïmeden, between the July 2003 and September 2004, have been presented. Measurements were taken with a first DIMM built by Moroccan astronomers. Median and mean seeing values of 0.84 arcsec and 0.92 arcsec, respectively, were achieved over the campaign. The results presented in this study have made it possible to enhance the qualification campaign of the Oukaimeden site started several years ago (Benkhaldoun 2000). The median seeing we found led us to conclude that the Oukaimeden is among the best observatory sites in the world as shown in Table 3. This study also shows that there is no obvious seasonal effect. It would be useful to continue measurements of the seeing over at least an additional year to verify this point. We also note a degradation of the seeing from the beginning to the end of this series of measurements. To investigate this phenomenon, we propose a study to include wind profile measurements which may influence the value of the seeing according to some studies.

Prospection of other sites in the Morrocan Atlas Mountains should also continue, since some may have even better seeing due to their location relative to the dominant wind flow.

Acknowledgements. We are grateful to the ESO which financially supported the campaigns at the Oukaïmeden site (Agreement No. 69651/ODG/02/9005/GWI). This work is also supported by the Morrocan research program (PARS No. 66/S DU/LPHEA).

It is a real pleasure to thank Marc SARAZIN from ESO and Jean VERNIN from Nice University for collaboration, conversation, and advice. Likewise, our sincere gratitude goes to the Omar Ouchaou, the site surveyor, and to all the staff members for their help and sympathy. We also want to give our thanks to George Jumper for his comments and careful reading of the manuscript. 


\section{References}

Aristidi, E., Agabi, A., Vernin, J., et al. 2002, Mem. S. A. It, 23, 73 Benkhaldoun, Z., Kadiri, S., Lazrek, M., \& Touma, H. 1991, Sol. Phys., 133, 61

Benkhaldoun, Z., Kadiri, S., Lazrek, M., \& Vernin, J. 1993, Experimental Astron., 2, 345

Benkhaldoun, Z. 1994, Ph.D. Thesis, Cadi Ayyad University, Marrakesh, Morocco

Benkhaldoun, Z. 2002, ASP Conf. Ser., 414, 266

Dierickx, P. J. 1992, Mod. Opt., 39, 569

Ehgamberdiev, S. A., Baijumanov, A. K., Ilyasov, S. P., et al. 2000, A\&AS, 145, 293

Hill, F., Fischer, G., Grier, J., et al. 1994a, Sol. Phys., 152(2), 321

Hill, F., Fischer, G., Forgach, S., et al. 1994b, Sol. Phys., 152(2), 351
Jabiri, A., Benkhaldoun, Z., Vernin, J., \& Muñoz-Tuñon, C. 2000, A\&A, 271, 284

Muñoz-Tuñón, C., Vernin, J., \& Varela, A. M. 1997, A\&A, 125, 183

National Astronomical Observatory of Japan 2003, http://www . naoj .org/Observing/Telescope/ Image/seeing.html

Roddier, F. 1981, Prog. Opt., 19, 281

Sarazin, M., \& Roddier, F. 1990, A\&A, 227, 294

Sarazin, M. 1999, http://www. eso.org/gen-fac/pubs/astclim/ paranal/seeing/singstory.html

Sarazin, M., Graham, E., Beniston, M., \& Riemer, M. 2003, SPIE, 4840, 291

Siher, E. A., \& Benkhaldoun, Z. 2004, New Astron., 9, 291

Tokovinin, A. 2002, PASP, 114, 1156

Vernin, J., \& Muñoz-Tuñón, C. 1994, A\&A, 284, 311

Ziad, A., Jabiri, A., Benkhaldoun, Z., et al. 2001, A\&A, 365, 324 